\title{
Effects of Particle Sizes on the Electromagnetic Property of Flaky FeSi Composites
}

\author{
Yonggang $X U^{\dagger}$, Liming YUAN, Jun CAI, Jianlu LV and Deyuan ZHANG \\ Bionic and Micro/Nano/Bio Manufacturing Technology Research Center, School of Mechanical Engineering and Au- \\ tomation, Beihang University, Beijing 100191, China
}

[Manuscript received 3 January 2013, in revised form 1 April 2013]

(C) The Chinese Society for Metals and Springer-Verlag Berlin Heidelberg

\begin{abstract}
Flaky FeSi absorbents with different size ranges were fabricated by sintering after mechanical milling process. $\mathrm{X}$-ray diffraction (XRD) was used to analyze the particle crystal grain structure. The complex permittivity and permeability of $\mathrm{FeSi} /$ paraffin composites were measured in frequency of 2-12 $\mathrm{GHz}$ using a vector network analyzer, and the DC electric conductivity was measured by the standard four-point contact method, then microwave reflection loss (RL) and shielding effectiveness (SE) were calculated. It was obtained that $\alpha$-Fe appeared in the super-lattice diffraction peaks in XRD pattern. As the particles size decreased, the permittivity decreased due to the inferior microwave electrical conductivity and dielectric loss tangent, while the permeability increased due to the decrease of diameter-thickness ratio, which could be demonstrated in the comparison between the experiment and calculation results. When thickness is $1 \mathrm{~mm}$, the composites with the smallest FeSi particles addition had a better absorbing property for the better impedance matching characteristic, and the minimum RL was $-7.9 \mathrm{~dB}$ at $4.6 \mathrm{GHz}$. While the composites with larger FeSi particles addition had an excellent shielding property due to the higher permittivity, the $\mathrm{SE}$ value ranged from $15 \mathrm{~dB}$ to $30 \mathrm{~dB}$ at the frequency band.
\end{abstract}

KEY WORDS: FeSi alloy; Absorbing property; Shielding property; Mechanical milling

\section{Introduction}

With the development of the wireless communication applications and wide use of the microwave devices over gigahertz $(\mathrm{GHz})$, electromagnetic interference (EMI) and electromagnetic compatibility (EMC) problems greatly interest researchers. Using microwave absorbing and EMI shielding materials is an effective way to overcome these problems due to the incident microwave attenuation and reflection in the materials ${ }^{[1,2]}$.

Nowadays, as a soft magnetic metal material, the FeSi particles have been widely used as the microwave absorbent due to the large values of saturation magnetization and Snoek's limit. The Snoek's limit could be described as $\left(\mu_{\mathrm{s}}-1\right) f_{\mathrm{r}}=\gamma M_{\mathrm{s}} /(3 \pi)^{[3,4]}$, where the right part of the expression is defined as Snoek's con-

† Corresponding author. Prof., Ph.D.; Tel: +86 010 82316603; E-mail address: xuyonggang221@163.com (Yonggang XU)

DOI: $10.1007 / \mathrm{s} 40195-013-0003-5$ stant, $\gamma$ denotes the gyromagnetic ratio and $M_{\mathrm{s}}$ is the saturation magnetization, $\mu_{\mathrm{s}}$ is the static permeability and $f_{\mathrm{r}}$ is the resonance frequency, both $\mu_{\mathrm{s}}$ and $f_{\mathrm{r}}$ could not be increased at the same time. Recently, in order to enhance the permeability, mechanical milling has been a conventional and effective way to shape the spherical particles to irregular or flaky shape. As the milled flaky particle thickness is lower than the skin depth, the eddy current effects can be suppressed effectively. The flaky absorbents have two types of magnetic anisotropy named easy-plane (parallel to the flaky particle plane) anisotropy and easy magnetization plane anisotropy. The parameters $\mu_{\mathrm{s}}$ and $f_{\mathrm{r}}$ for easy-plane anisotropy materials can be obtained ${ }^{[5,6]}$ : $\left(\mu_{\mathrm{s}}-1\right) f_{\mathrm{r}}=\gamma M_{\mathrm{s}} \sqrt{H_{\mathrm{ha}} / H_{\mathrm{ea}}} /(3 \pi)$, where $H_{\mathrm{ha}}$ and $H_{\mathrm{ea}}$ denote the effective anisotropy field when the magnetization deviates from the easy axis in the hard-plane (perpendicular to the easy-plane) and in the easyplane respectively $\left(H_{\mathrm{ha}}>H_{\mathrm{ea}}\right)$, so the Snoek's limit of the flaky particles was larger than that of spherical ones ${ }^{[7,8]}$. As a result, the absorption property of 


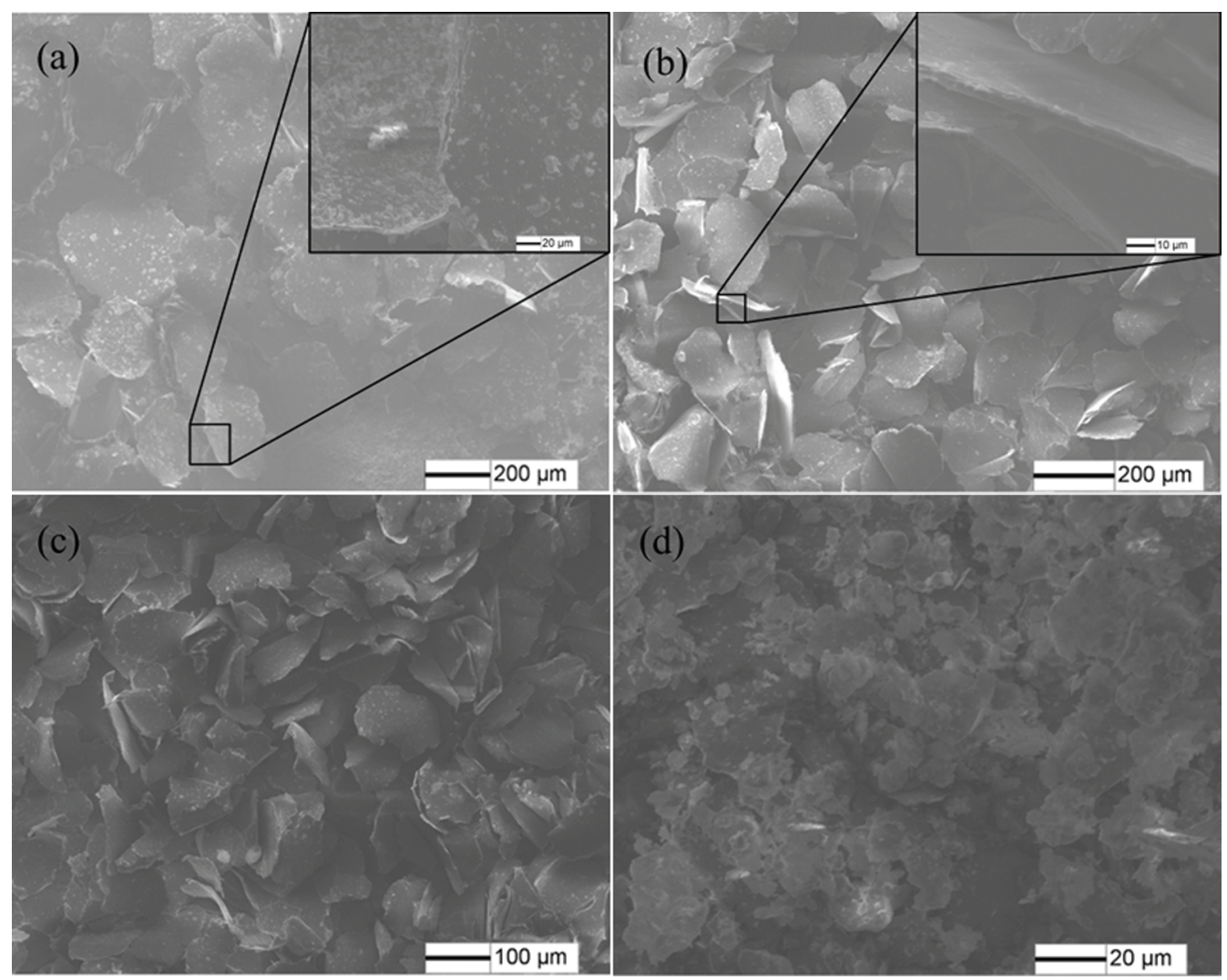

Fig. 1 SEM micrographs of flaky FeSi powders with different diameter ranges: (a) 150-250 $\mu$ m; (b) $75-150 \mu \mathrm{m}$; (c) $40-75 \mu \mathrm{m}$; (d) less than $40 \mu \mathrm{m}$

absorbing materials filled with flaky $\mathrm{FeSi}$ particles could be enhanced than those with spherical FeSi as the filler ${ }^{[8,9]}$. For example, as the complex permeability increased, the composite thickness could be much thinner than that with spherical FeSi as the filler to achieve the required higher absorption ratio and wider absorption frequency band. Rozanov et al. ${ }^{[10,11]}$ have proposed a new mixing rule to calculate the permeability of composites with the absorbent addition, in which the effect of the inclusions shape distribution was studied. However, the particle size of absorbents was just taken into account on the spherical shape due to their simple isotropic property ${ }^{[12]}$, the influence of flaky particle size on the electromagnetic property and the optimum particle size of flaky FeSi was rarely reported.

The objective of this work is to analyze the particle size effect on the microwave absorbing/shielding materials using the commercial FeSi as the absorbents. The mainly focus was on the permittivity and permeability of paraffin composites added flaky FeSi with different size. Finally, the absorbing and shielding property of each composite would be discussed in order to obtain an optimum particle sizes.

\section{Materials and Experiment}

\subsection{Materials preparation}

Flaky FeSi (93.5 wt.\% Fe-6.5 wt.\% Si) particles were prepared by means of the mechanical milling method using the raw spherical FeSi particles with an average diameter $75 \mu \mathrm{m}$ and were supplied by Changsha Hualiu Power Co. Ltd, China. Pre-grinded FeSi particles, zirconia balls and n-hexane were introduced to a zirconia jar. The mass ratios of zirconia ball, $\mathrm{FeSi}$ particles and n-hexane were 20: 1: 1 . The mechanical milling process was carried out in a SP2 planetary ball milling machine for $8 \mathrm{~h}$ with the revolving speed of $500 \mathrm{r} / \mathrm{min}$. Then the flaky particles were sieved using three sieves of different meshes, the diameters of the particles were distributed in the size range of less than $40 \mu \mathrm{m}, 40-75 \mu \mathrm{m}, 75-150 \mu \mathrm{m}$ and 150-250 $\mu \mathrm{m}$. Fig. 1 shows the SEM micrographs of sieved flaky FeSi particles with different sizes, and most of the particles are flaky and the average thickness was $2 \mu \mathrm{m}$. Then flaky FeSi was added into the paraffin matrix with 30 vol.\% content by a mechanical dispersion method. The testing composites for complex permittivity and permeability measurement were die-pressed to a toroid shape with an outer diameter of $7.0 \mathrm{~mm}$, inner diameter of $3.04 \mathrm{~mm}$ and thickness of $2 \mathrm{~mm}$.

\subsection{Testing}

The morphology of the absorbents was observed by scanning electron microscopy (SEM CamScan CS3400). The phases of the powders were estimated 
by X-ray diffraction (XRD, D/MX 2200) using $\mathrm{Cu} K_{\alpha}$ radiation (wavelength is $0.154 \mathrm{~nm}$ ), scan step was $0.02^{\circ} / \mathrm{s}$. The DC electric conductivity was measured on pressed rectangular of the composites prepared at room temperature by the standard four-point contact method. The permittivity and permeability of the absorbers were measured using the transmission method with an AV3627 vector network analyzer in a frequency range of 2-12 GHz. Then the RL and SE could be calculated to evaluate the absorbing and shielding property using the tested EM parameters. For an absorbing material, the RL of normal incident EM wave at the absorber surface can be defined as the ratio of reflected power to incident power by testing the absorbers backed by a perfect electronic conductor, the shielding effectiveness (SE) can be defined as the ratio of the transmitted power to incident power. They can be represented by the following equations ${ }^{[13]}$ :

$$
\begin{gathered}
\Gamma_{0}=\left(\sqrt{\mu_{\mathrm{r}} / \varepsilon_{\mathrm{r}}}-1\right) /\left(\sqrt{\mu_{\mathrm{r}} / \varepsilon_{\mathrm{r}}}+1\right) \\
Z_{\text {in }}=Z_{0} \sqrt{\mu_{\mathrm{r}} / \varepsilon_{\mathrm{r}}} \tanh \left(2 \pi d j \sqrt{\mu_{\mathrm{r}} / \varepsilon_{\mathrm{r}}} / \lambda\right) \\
T=\exp \left(-j \frac{2 \pi d}{\lambda} \sqrt{\mu_{\mathrm{r}} \cdot \varepsilon_{\mathrm{r}}}\right) \\
\mathrm{RL}=20 \lg \left|\left(Z_{\mathrm{in}}-Z_{0}\right) /\left(Z_{\mathrm{in}}+Z_{0}\right)\right| \\
\mathrm{SE}=-20 \lg \left|\left(1-\Gamma_{0}^{2}\right) \mathrm{T} /\left(1-\mathrm{T}^{2} \Gamma_{0}^{2}\right)\right|
\end{gathered}
$$

where $Z_{\text {in }}$ is the normalized input impedance of the absorbing material, $Z_{0}=120 \pi$ is the intrinsic impedance of free space, $\Gamma_{0}$ is the reflection coefficient, $T$ is the transmission coefficient, $\varepsilon_{\mathrm{r}}$ and $\mu_{\mathrm{r}}$ are complex permittivity and complex permeability of the absorbing material, respectively, $\lambda$ is the wavelength of the microwave, and $d$ is the thickness of the absorbing material.

\section{Results and Discussion}

\subsection{XRD patterns of the FeSi particle}

Fig. 2 shows the XRD patterns of the flaky and spherical FeSi powders. The XRD spectrum for the powders indicated the reflection corresponds to distinct body-centered cubic (bcc) Fe metals. The reflection peaks (110), (200) and (211) of bcc Fe are observed from the two particles. As the powder was milled, it was noted that the B2 superlattice phase became smooth, the characteristic peaks became broadened and the intensity of their diffraction pattern increased which meant that the amorphous tendency of FeSi particles decreased. Furthermore, an obvious broadening of the Si diffraction line was also found at the angle range of $26^{\circ}-27^{\circ}$, it could be concluded that $\mathrm{Si}$ atoms dissolve in the Fe lattice entirely, which resulted in the formation of face-centered cubic (fcc) Fe solid solution. It was noticeable that although the super-lattice diffraction peaks of $\alpha$-Fe appeared, the stoichiometric calculations were very complex including $\mathrm{Fe}_{3} \mathrm{Si}, \mathrm{Fe}_{5} \mathrm{Si}_{3}, \mathrm{FeSi}$ or $\mathrm{FeSi}_{2}^{[8,9,14]}$. It was difficult

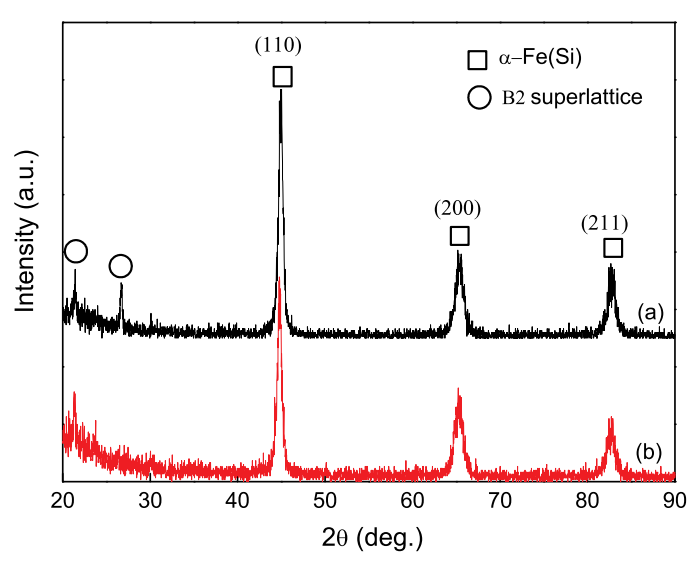

Fig. 2 XRD patterns of the flaky FeSi (a) and spherical FeSi (b) particles

to distinguish the differences between them for they were similar and crossed together in the composites. During the mechanical milling process, FeSi powders adhered on the $\mathrm{ZrO}_{2}$ balls, collision and milling occurred between the balls and inner wall of the $\mathrm{ZrO}_{2}$ vial. The spherical powders would be deformed to be smaller and become flaky shape, and later the flaky particles were overlapped and the cold weld existed together, as a result, the flaky powders with various particle sizes were produced.

\subsection{Complex permittivity and permeability of the com- posites}

The EM parameters including complex permittivity $(\varepsilon)$ and complex permeability $(\mu)$ of each composite dependent on the frequency are shown in Fig. 3. First, the composite added flaky FeSi with average diameter 150-250 $\mu \mathrm{m}$ had a larger $\varepsilon$ than other FeSi particles, the real part permittivity $\left(\varepsilon^{\prime}\right)$ decreased from 196 to 118 and the imaginary part permittivity $\left(\varepsilon^{\prime \prime}\right)$ decreased from 140 to 90 . As the particle size decreased, the value of $\varepsilon^{\prime}$ and $\varepsilon^{\prime \prime}$ decreased dramatically. The flaky FeSi with average diameter $40-75 \mu \mathrm{m}$ had the smallest $\varepsilon$, the average $\varepsilon^{\prime}$ was 50 and $\varepsilon^{\prime \prime}$ was about 5 . While the real part permeability $\left(\mu^{\prime}\right)$ of each flaky FeSi decreased monotonously from $2 \mathrm{GHz}$ to $12 \mathrm{GHz}$, and imaginary part permeability $\left(\mu^{\prime \prime}\right)$ was also decreased in the range of $2-12 \mathrm{GHz}$ except the particles with average diameter less than $40 \mu \mathrm{m}$. As the particle diameter was larger than $40 \mu \mathrm{m}$, the changes of $\mu^{\prime}$ and $\mu^{\prime \prime}$ were less than 0.2 and 0.3 , respectively. While $\mu^{\prime}$ and $\mu^{\prime \prime}$ of the particles smaller than $40 \mu \mathrm{m}$ were much larger than that of other samples. The maximum of $\mu^{\prime \prime}$ of the particles smaller than $40 \mu \mathrm{m}$ was achieved as 2.65 at $4.9 \mathrm{GHz}$. The changing tendency of $\mu^{\prime}$ and $\mu^{\prime \prime}$ was owing to the particle size.

The DC conductivity $(\sigma)$ of each composite is listed in Table 1. It was found that the conductivity of the composites increased with the particles size increased. In this work, the volume content of $\mathrm{FeSi}$ 

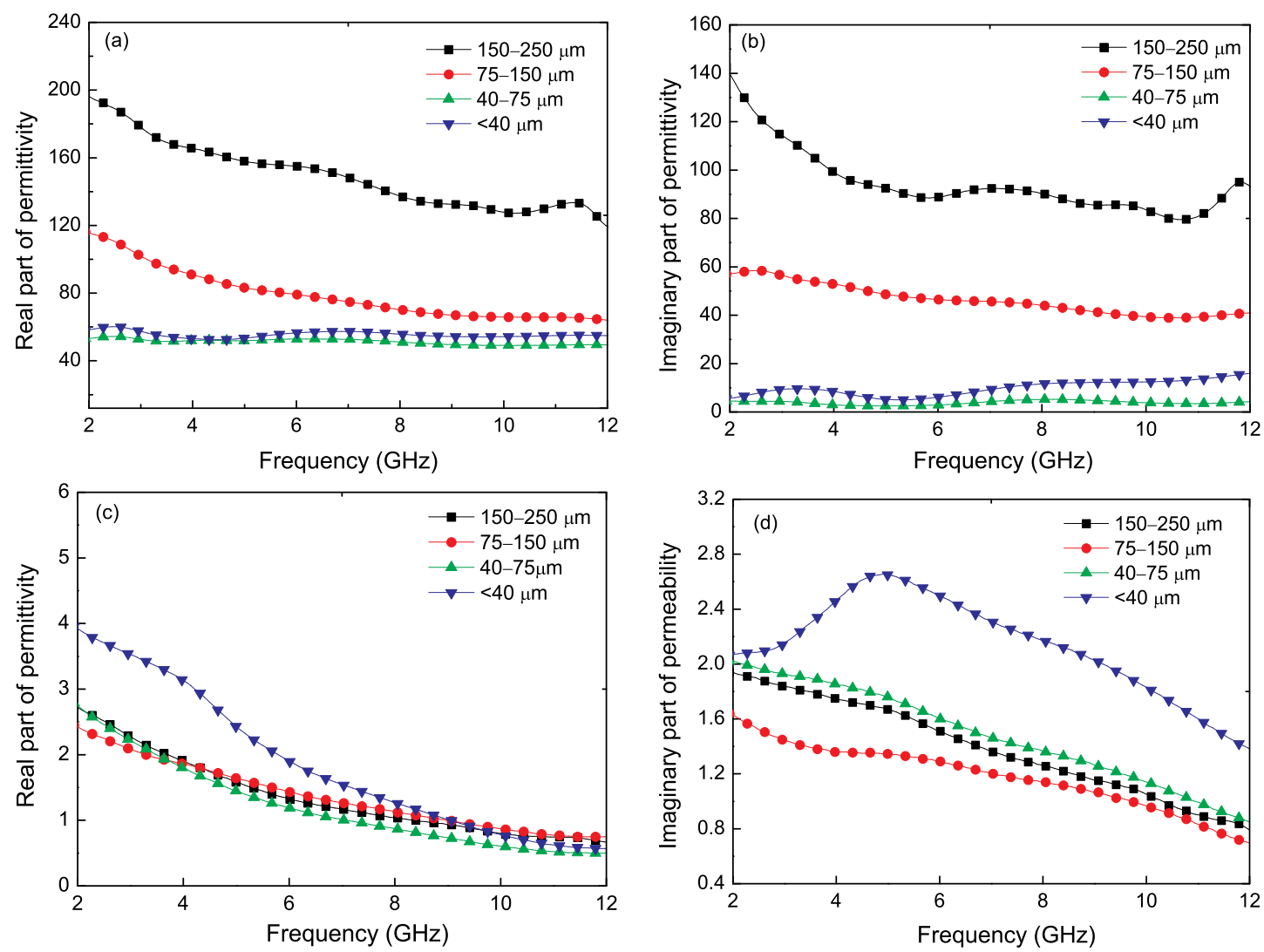

Fig. 3 Electromagnetic parameters of the composites in the frequency range of 2-12GHz: (a) real part of permittivity; (b) imaginary part of permittivity; (c) real part of permeability; (d) imaginary part of permeability

Table 1 Conductivities $(\sigma)$ of the composites with different size FeSi

\begin{tabular}{cc}
\hline FeSi particle size & Conductivity $\sigma(\mathrm{S} / \mathrm{cm})$ \\
\hline $150-250 \mu \mathrm{m}$ & 0.05 \\
$75-150 \mu \mathrm{m}$ & 0.03 \\
$40-75 \mu \mathrm{m}$ & 0.02 \\
$<40 \mu \mathrm{m}$ & 0.01 \\
\hline
\end{tabular}

was close to the percolate threshold. The real part of permittivity was related to the microwave electrical conductivity, which means that a larger conductivity often resulted in a larger real part permittivity. The conductivity mechanism could be interpreted by two ways: the first was concerned with the volume content and the intrinsic conductivity of the particles; the other was the gaps between the absorbents, the small gaps between particles could make the conductivity network formed easily. Among the four FeSi/paraffin composites, the volume content and intrinsic conductivity of the particles were the same, while, if the particle size was larger, the particle shape aspect ratio or the diameter-thickness ratio was larger also. According to the conductive theory, in the composites filled with larger particles, the larger flakes could be seen as aggregating particles of the oriented and connected smaller flakes. The conductive network could be improved compared with the composite with smaller particles. It can be explained by $V_{\mathrm{ex}}=v_{\mathrm{c}} e^{[15,16]}$, where $V_{\text {ex }}$ is the critical volume excluded, $e$ is the diameterthickness ratio, $v_{\mathrm{c}}$ is the percolation threshold. If the larger particles had a larger $e$, as well as $V_{\mathrm{ex}}$, so it made a larger probability of the particle contact, the conductive network could be improved compared with the composite with smaller particles. As a result, the composites filled with larger absorbent had a higher conductivity and real part of permittivity. In addition, the imaginary part of permittivity was determined by the real part of permittivity and the dielectric loss tangent. The dielectric loss mechanism mainly includes the interfacial polarization and orientation polarization. Among the four composites the orientation polarization of flaky FeSi could be considered nearly the same due to the random dispersion of the particles and the same fabrication process. While the interfacial polarization of the composites could be enhanced as particles of higher aspect ratio were added due to the large contact probability between the particles. Therefore, the particles with large aspect ratio or particle size had a large dielectric permittivity.

Meanwhile, the permeability of the composites could be explained by the Maxwell-Garnett mixing rule. As the $\mathrm{FeSi} /$ paraffin absorbers with arbitrary orientation and flakes are more densely packed, the effective permeability is isotropic and given as ${ }^{[17,18]}$ : 


$$
\begin{gathered}
\mu_{\mathrm{eff}}=\mu_{\mathrm{m}}+v \mu_{\mathrm{a}} \frac{\mu_{\mathrm{i}}-\mu_{\mathrm{m}}}{\mu_{\mathrm{a}}+\left(1-v / v_{\mathrm{c}}\right) n\left(\mu_{\mathrm{i}}-\mu_{\mathrm{m}}\right)} \\
\mu_{\mathrm{a}}=\mu_{\mathrm{m}}+a\left(\mu_{\mathrm{eff}}-\mu_{\mathrm{m}}\right)
\end{gathered}
$$

where $\mu_{\mathrm{i}}$ and $\mu_{\mathrm{m}}$ are the permeability of inclusion and matrix respectively, $\mu_{\mathrm{m}}=1 ; \mu_{\mathrm{a}}$ was apparent permeability; $a$ is a pending parameter between 0 and $1 ; n$ is the averaged shape factor of fillers, $n=1 / 3$ as the spherical particle was the filler, $n \approx 1 /\left(1+1.6 e+0.4 / e^{2}\right)$ as the flaky particle was the filler; $v$ is the volume fraction. The average $e$ of the four particles is set as $100,50,25$ and 10, as a result, $n$ of the four particles is about $0.98,0.97,0.94$ and 0.86 , respectively. The Maxwell-Garnett mixing rule predicts that the percolation threshold of the conducting constituent in the host matrix is $v_{\mathrm{c}}=1$, but the experiment results are not always in agreement with them for the percolation threshold mainly depending on average particle size and distribution.

Moreover, in order to establish the intrinsic permeability of the magnetic materials dependent on the frequency, the Gilbert-Landau-Lifshitz equation was proposed, in which the magnetization $\vec{M}$ under a magnetic field $\vec{H}$ was expressed as follow ${ }^{[19]}$ :

$$
\frac{\mathrm{d} \vec{M}}{\mathrm{~d} t}=\gamma(\vec{M} \times \vec{H})+\frac{\alpha}{M} \vec{M} \times \frac{\mathrm{d} \vec{M}}{\mathrm{~d} t}
$$

where $\alpha$ is the damping coefficient, and $\gamma$ is the gyromagnetic ratio. Later, Wallace ${ }^{[19]}$ developed a formula of intrinsic permeability based on the above equation, in which $\mu_{\mathrm{i}}$ can be obtained as:

$$
\mu_{\mathrm{i}}=1+\frac{\left(4 \pi M_{\mathrm{s}}\right)^{2}}{\left(4 \pi M_{\mathrm{s}}\right) H_{\mathrm{a}}-(f / 2.8)^{2}+j \alpha\left(4 \pi M_{\mathrm{s}}\right)(f / 2.8)}
$$

where $M_{\mathrm{s}}$ is the saturation magnetization, $H_{\mathrm{a}}$ is the magnetic anisotropy field, and $f$ is the frequency. In order to simplify the problem, the effect of the shape anisotropic property of the FeSi particles was ignored in Wallace's equation, and the following observed result could verify it. So the EM parameters in this equation could be used to calculate the intrinsic permeability of the flaky FeSi, and then the effective permeability of each composite could be calculated in Maxwell-Garnett mixing rule. In order to obtain the effective permeability, the pending parameters including $a, M_{\mathrm{s}}, H_{\mathrm{a}}, \alpha$ and $v_{\mathrm{c}}$ were often fitted. However the calculation process was always complicated and timewasting. Here, we use an interpolation method to calculate functions on the intrinsic permeability and shape factor according to the relationship between the permeability and the magnetic susceptibility. For the spherical particle material, $\mu_{\mathrm{i}}=1+\chi$, where $\chi$ is the magnetic susceptibility of the particles. And as the flaky particle was the filler, $\mu_{\mathrm{i}}=1+\frac{1}{2} \chi_{\mathrm{p}}+\frac{1}{2} \chi_{\mathrm{v}} \cos ^{2} \theta$, where $\chi_{\mathrm{p}}$ and $\chi_{\mathrm{v}}$ are the magnetic susceptibility along the axis parallel and vertical to the flaky plane respectively, $\theta$ is the average angle of particle flaky plane

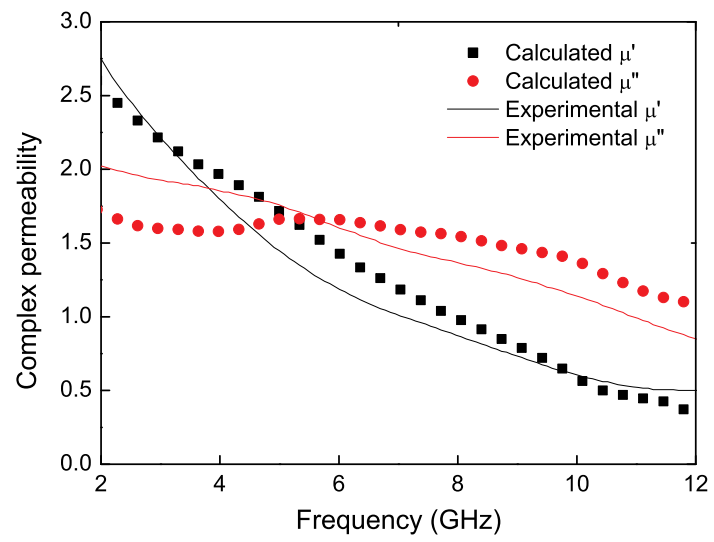

Fig. 4 Comparison between the experimental and calculated permeability of the composite with $75-$ $150 \mu \mathrm{m} \mathrm{FeSi}$

and the testing sample horizontal plane, and as the flaky particles were randomly dispersed, the value of $\cos ^{2} \theta$ was $1 / 3^{[20]}$. In this paper, $\chi_{\mathrm{p}}$ was considered as the same for the flakes were fabricated in the same process and had the same average thickness, and the $\chi_{\mathrm{v}}$ had a linear relation to the averaged shape factor given the shape factor didn't change obviously. Then the obtained permeability of the sample was used to calculate the intrinsic permeability of the flaky FeSi as the pending parameters $a$ and $v_{\mathrm{c}}$ could be fitted.

The comparison between the experiment and the calculation results on the composite permeability with 30 vol.\% FeSi $(75-150 \mu \mathrm{m})$ added is shown in Fig. 4. The fitting parameters were $a=0.3$ and $v_{\mathrm{c}}=0.5$, respectively. As it is shown in Fig. 4, the fitting results have an excellent agreement with the experiment data over the whole frequency band. This demonstrated that the permeability of the composites was greatly determined by the shape factor of fillers, for the above calculation method was validated in the early research ${ }^{[21]}$.

\subsection{Absorbing and shielding properties of the compos- ites}

The RL and SE of the four samples are shown in Fig. 5 as a function of frequency in the range of 2-12 GHz. It was obviously obtained that as the thickness was constant $1 \mathrm{~mm}$, the matching frequency of composites added the largest particles was the lowest $(3.0 \mathrm{GHz})$. It was in fairly accord with the equation $^{[20]}: f_{\mathrm{m}}=c\left(1+\tan ^{2} \delta_{\mu} / 8\right) /\left(4 d_{\mathrm{m}} \sqrt{|\mu \varepsilon|}\right.$, where $c$ denotes the velocity of the light, $\tan \delta_{\mathrm{u}}$ denotes the magnetic loss tangents, $d_{\mathrm{m}}$ is the matching thickness and $|\mu \varepsilon|$ is the matching frequency. As $d_{\mathrm{m}}$ was a constant, the composites which had the largest $|\mu \varepsilon|$ would result in the lowest $f_{\mathrm{m}}$. So, the composites added 150-250 $\mu \mathrm{m}$ FeSi had the lowest matching frequency caused by the largest permittivity and $|\mu \varepsilon|$, and the $\mathrm{RL}$ value was the largest which means that the absorbing property of the composites was worst of the 

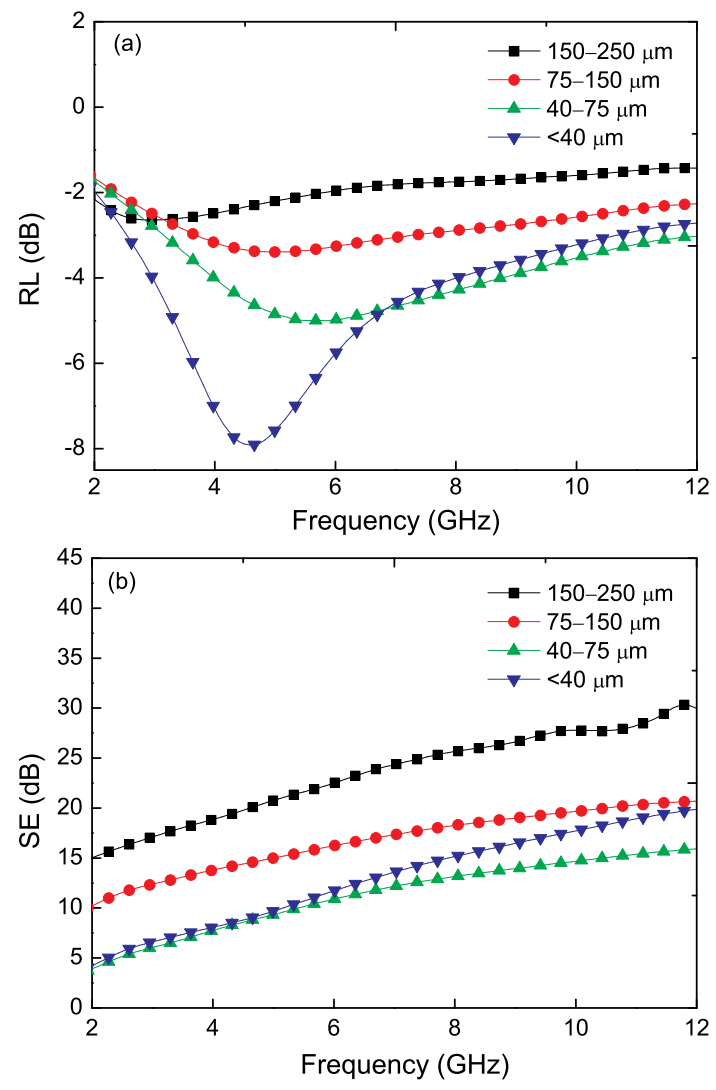

Fig. 5 Absorbing and shielding properties of the $1 \mathrm{~mm}$ thick composites with different sizes FeSi: (a) RL of samples; (b) SE of samples

all. While the composites added the smallest FeSi particles had the least RL, and the minimum RL was $-7.9 \mathrm{~dB}$ at $4.6 \mathrm{GHz}$. It was attributed to the complex permittivity and complex permeability, for the two parameters determined the microwave absorbing and shielding property of the composite and reflected the dielectric loss and magnetic loss of the materials. The absorbing materials with FeSi as the filler had a much larger permittivity than the permeability. In order to increase the absorption ratio of the composites, the permeability should be raised and the permittivity reduced according to the impedance matching characteristics. So decreasing the particle size had a positive effect on enhancing the absorbing property.

Meanwhile, the SE of each composite increased as the frequency increased, the largest $\mathrm{SE}$ value (nearly $30 \mathrm{~dB}$ ) could be obtained at $12 \mathrm{GHz}$ as the 150 $250 \mu \mathrm{m} \mathrm{FeSi}$ was the filler. As the particles size decreased, the SE began to decrease and then changed slightly. The SE was mainly determined by the conductivity of the composites, as well as the complex permittivity. For the formation of more networks of conductive filler and larger source of free electrons in the material could interact with incident electromagnetic wave ${ }^{[23]}$. So the composites with largest FeSi particles added had the largest permittivity which resulted in the best shielding property. In addition, the total SE can be represented by the sum of contribu-

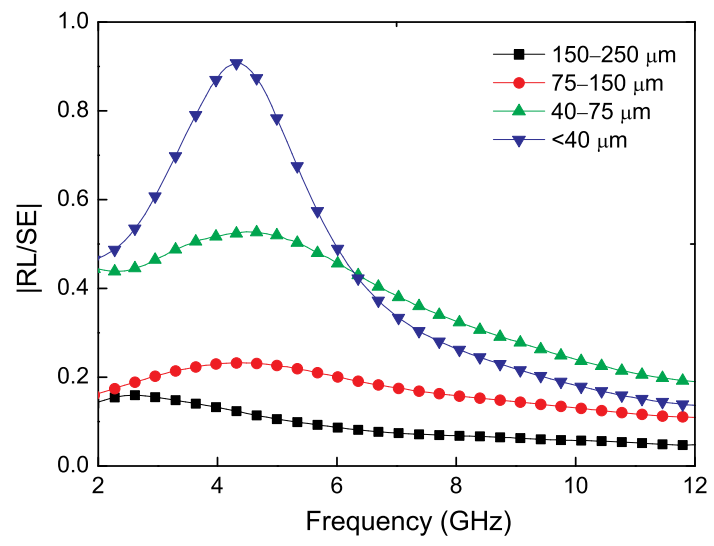

Fig. 6 Absolute value of the ratio of RL to SE of $1 \mathrm{~mm}$ thick composites with different sizes FeSi in the frequency range of $2-12 \mathrm{GHz}$

tions from absorption loss, reflection loss and multiple reflections, while the absolute RL is mainly represented by the absorption loss during tested by a metal substrate. In this work, the absorption loss of the absorbers is much less than the $\mathrm{SE}$ value at the most of frequency. Fig. 6 showed the ratio of $|\mathrm{RL} / \mathrm{SE}|$ of each sample of thickness $1 \mathrm{~mm}$ as a function of frequency. It could be obtained that the $|\mathrm{RL}|$ value was much less than SE except the composites with FeSi smaller than $40 \mu \mathrm{m}$. For the other three composites, it could be concluded that the reflection of the microwave occupied a dominant part of the shielding property $(|\mathrm{RL} / \mathrm{SE}|$ was almost less than $0.2,0.25$ and 0.5 , respectively). While $|\mathrm{RL} / \mathrm{SE}|$ was larger than 0.5 of the composites with FeSi smaller than $40 \mu \mathrm{m}$ in the frequency range of 2.4-6.0 GHz, and the maximum ratio was 0.90 at $4.3 \mathrm{GHz}$, which means that the absorption of the microwave mainly dominated the shielding property at this frequency band. However, how to simultaneously improve the absorbing/shielding property of $\mathrm{FeSi} /$ paraffin composites with an optimum particle size will be established in a further investigation.

\section{Conclusion}

FeSi powders with different size ranges were fabricated by mechanical milling method and the electromagnetic and microwave absorbing/shielding properties were studied. The particle size had different influences on the absorbing and shielding properties of the FeSi/paraffin composites. The smaller particle size made a smaller permittivity due to the inferior electrical conductivity and dielectric loss tangent, and resulted in a larger permeability due to the smaller average shape factor. The composites added the smallest FeSi particles had the best absorbing property and the minimum RL was $-7.9 \mathrm{~dB}$ at $4.6 \mathrm{GHz}$ with thickness of $1 \mathrm{~mm}$, while the composites added the largest FeSi particles had the best shielding property with the SE value 15-30 dB. Therefore, in order to obtain an ex- 
cellent absorbing/shielding composite, absorbent size range should be taken into account.

\section{Acknowledgements}

This work was supported by the National Natural Science Foundation of China (No. 51275025), High Technology Research and Development Program of China (No. 2009AA043804), the Foundation for the Author of National Excellent Doctoral Dissertation of PR China (No. 2007B32) and the Innovation Foundation of BUAA for PhD Graduates.

\section{REFERENCES}

[1] M. Itoh, J.R. Liu, T. Horikawa and K.I. Machida, J. Alloys Compd. 408-412 (2006) 1400.

[2] Y.C. Qing, W.C. Zhou, F. Luo and D.M. Zhu, J. Magn. Magn. Mater. 321 (2009) 25.

[3] J.L. Snoek, Physica 14 (1948) 207.

[4] R. Lebourgeois, C.L. Fur, M. Labeyrie, M. Pate and J.P. Ganne, J. Magn. Magn. Mater. 160 (1996) 329.

[5] T.L. Gilbert, IEEE Trans. Magn. 40 (2004) 3443.

[6] D.S. Xue, F.S. Li, X.L. Fan and F.S. Wen, Chin. Phys. Lett. 25 (2008) 4120.

[7] R. Han, L. Qian, T. Wang and F.S. Li, J. Alloys Compd. 509 (2011) 2734.

[8] T.D. Zhou, P.H. Zhou, D.F. Liang and L.J. Deng, J. Alloys Compd. 484 (2009) 545.
[9] G.K. Tian and X.F. Bi, J. Alloys Compd. 502 (2010) 1.

[10] K.N. Rozanov, A.V. Osipov, D.A. Petrov, S.N Starostenko and E.P. Yelsukov, J. Magn. Magn. Mater. 321 (2009) 738.

[11] K.N. Rozanov, M.Y. Koledintseva and J.L. Drewniak, J. Magn. Magn. Mater. 324 (2012) 1063.

[12] L.D. Liu, Y.P. Duan, J.B. Guo, L.Y. Chen and S.H Liu, Phys. B 406 (2011) 2261.

[13] J. Joo and A.J. Epstein, Appl. Phys. Lett. 65 (1994) 2278.

[14] G.Z. Xie, L.K. Yuan, P. Wang, B.S. Zhang, P.H. Lin and H.X. Lu, J. Non-Cryst. Solids 356 (2010) 83.

[15] A. Lonjon, P. Demont, E. Dantras and C. Lacabanne, J. Non-Cryst. Solids 358 (2012) 3074.

[16] I. Balberg, C.H. Anderson, S. Alexander and N. Wagner, Phys. Rev. B 30 (1984) 3933.

[17] D.T. Zimmerman, J.D. Cardellino, K.T. Cravener, K.R. Feather, N.M. Miskovsky and G.J. Weisel, Appl Phys. Lett. 93 (2008) 214103.

[18] A.H. Sihvola, IEEE Trans. Geosci. Remote. Sens. 27 (1989) 403

[19] J. L. Wallace, IEEE Trans. Magn. 29 (1993) 4209.

[20] T. Liu, P.H. Zhou, J.L. Xie and L.J. Deng, J. Magn. Magn. Mater. 324 (2012) 519.

[21] L.Z. Wu, J. Ding, H.B. Jiang, L.F. Chen and C.K. Ong, J. Magn. Magn. Mater. 285 (2005) 233.

[22] S.S. Kim, S.B. Jo, K.I. Gueon, K.K. Choi, J.M. Kim and K.S. Churn, IEEE Trans. Magn. 27(1991) 5462.

[23] M. Arjmand, M. Mehdi, A.G. Genaro, S. Park and U. Sundararaj, Carbon 49 (2011) 3430. 